\title{
Proposed mechanism for cyst formation and enlargement following Gamma Knife Surgery for arteriovenous malformations
}

\author{
Clinical article
}

\author{
Takashi Shuto, M.D., Makoto Ohtake, M.D., and Shigeo Matsunaga, M.D. \\ Department of Neurosurgery, Yokohama Rosai Hospital, Yokohama, Kanagawa, Japan
}

\begin{abstract}
Object. The authors retrospectively studied the mechanism of cyst formation and enlargement after Gamma Knife surgery (GKS) for arteriovenous malformations (AVMs).

Methods. Eighteen patients in whom cyst formation developed following GKS for AVM were retrospectively identified among 775 patients who underwent GKS for AVM at Yokohama Rosai Hospital. The study group was composed of 12 male and 6 female patients ranging in age from 17 to 47 years.

Results. Chronic encapsulated expanding hematoma was associated with the cyst in 5 patients. The AVM nidus volume at the time of GKS ranged from 1.9 to $36 \mathrm{~cm}^{3}$, and the prescription radiation dose was 18-25 Gy. Complete obliteration of the AVM nidus was obtained in 13 patients and partial obliteration in 5 patients. Cyst formation was detected between 2.6 and 15 years after GKS. Craniotomy was performed in 10 patients, including 2 patients in whom the incompletely obliterated nidus was removed at the same time, and an Ommaya reservoir was placed in 2 patients. Spontaneous regression of the cyst was observed in 1 patient. Serial MR imaging was performed in the other patients because the size of the cyst was stable or the lesion was asymptomatic. Histological examination of the cyst wall revealed linear hemosiderin deposits with gliosis. The nodular lesion, which was enhanced on MR images, contained granulation tissue with chronic hemorrhage from newly developed capillary vessels.

Conclusions. Cysts developing after GKS for AVM enlarge mainly due to repeated minor hemorrhages from a reddish nodular angiomatous lesion that develops within an adjacent brain area. Thus, the optimal treatment is wide opening of the cyst with removal of the associated angiomatous lesion by craniotomy.

(http://thejns.org/doi/abs/10.3171/2012.6.GKS12318)
\end{abstract}

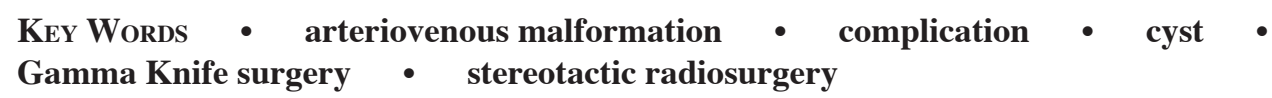

$\mathrm{G}$ AMMA knife surgery is widely used as an effective initial or adjuvant treatment of cerebral AVMs. ${ }^{12,15,18}$ Unfortunately, GKS for AVMs may be followed by various complications such as radiationinduced edema, ${ }^{1-3}$ cyst formation, ${ }^{7,16,20}$ chronic encapsulated expanding hematoma, ${ }^{11,13,14}$ and radiation-induced tumor. ${ }^{8}$ Cyst formation is the most common complication, but the mechanisms of cyst formation and enlargement remain unclear. This complication is clinically important because surgical treatment is required in some cases. ${ }^{19}$ The incidence of cyst formation is $1.6 \%$ of all treated cases, but it is $3.6 \%$ in patients whose follow-up extends longer than 5 years after GKS, indicating cyst formation is more likely to occur over longer periods. ${ }^{16}$

In the present study we retrospectively analyzed cases in which cyst formation occurred after GKS for treat-

Abbreviations used in this paper; $\mathrm{AVM}=$ arteriovenous malformation; GKS = Gamma Knife surgery. ment of an AVM so that we could evaluate the mechanisms of cyst formation and enlargement.

\section{Methods}

Twelve male and 6 female patients, ranging in age from 17 to 47 years (mean 27.3 years), were retrospectively identified among 775 patients who underwent GKS for an AVM at our hospital (Table 1). Gamma Knife surgery was performed with the aid of a Leksell gamma unit (model B; Elekta Instruments, Inc., Norcross, GA) and Gamma Plan treatment software (Elekta AB, Stockholm, Sweden) or KULA (Elekta AB). Not all patients who underwent GKS at our hospital underwent follow-up with us; thus, the exact occurrence rate of late complications was unclear. In our retrospective analysis we examined the calculated volume of the AVM nidus, the prescription radiation dose delivered to the nidus margin, the timing of changes on neuroimages, follow-up images of cysts, in- 


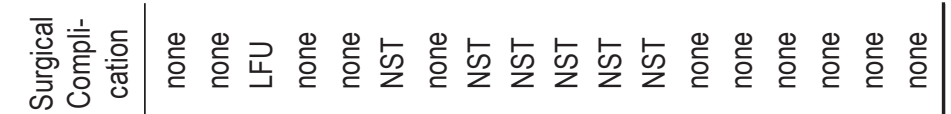

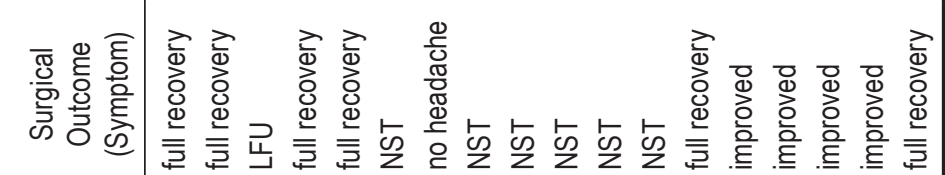

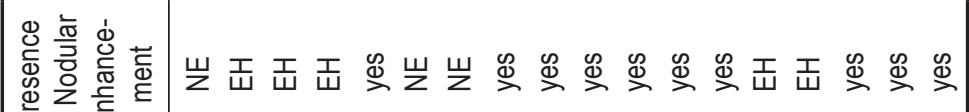
这说焉

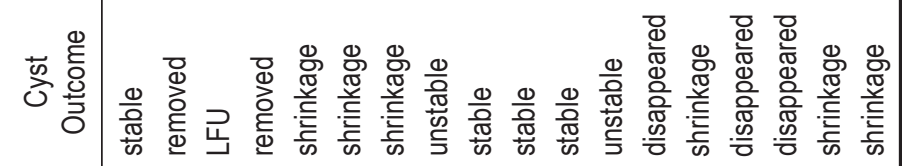

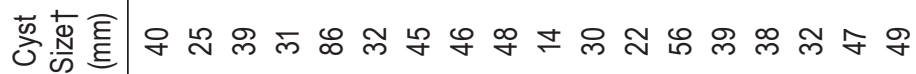

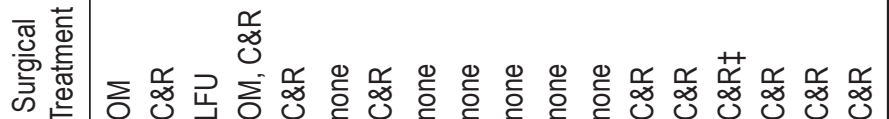

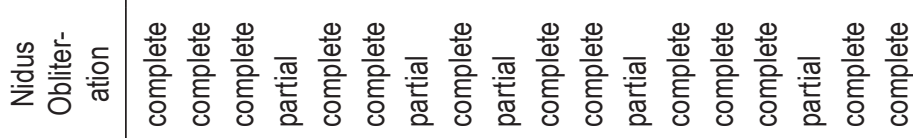

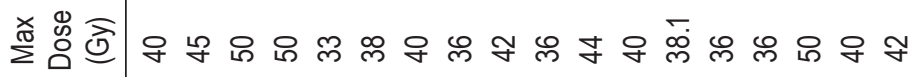

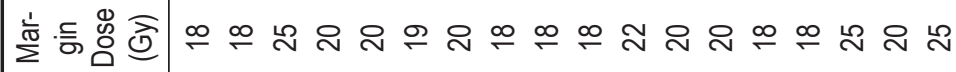

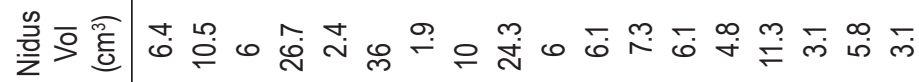

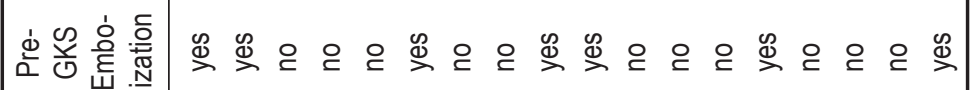

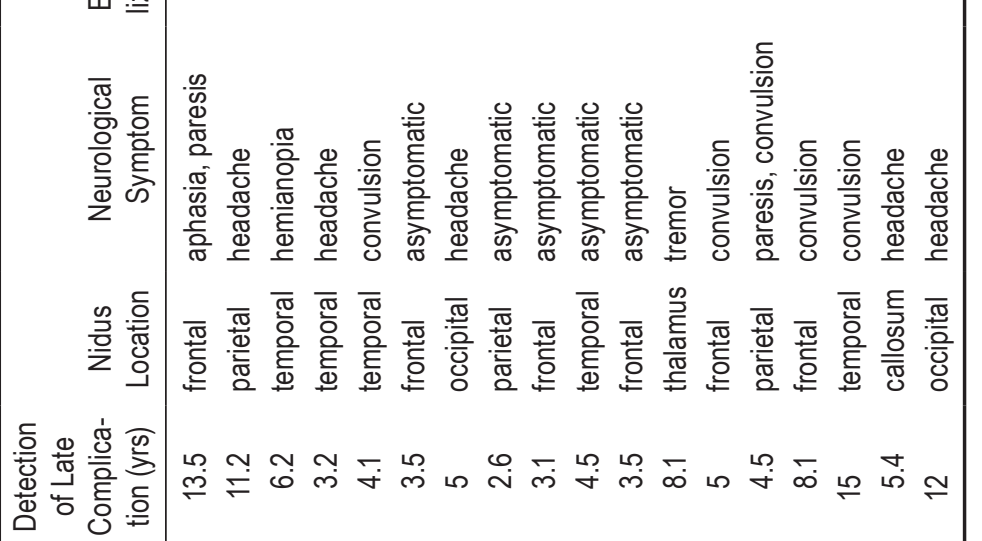

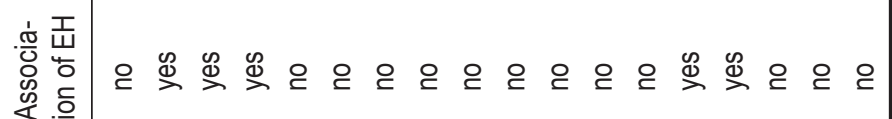

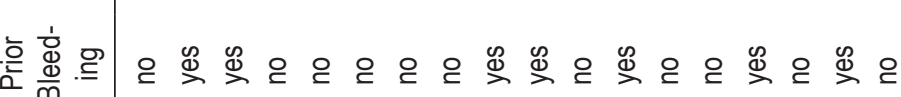

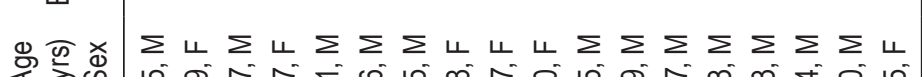

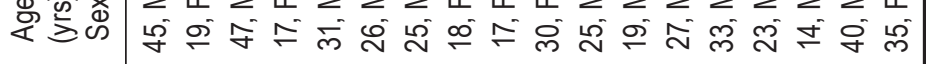
惫 $\mid$ -

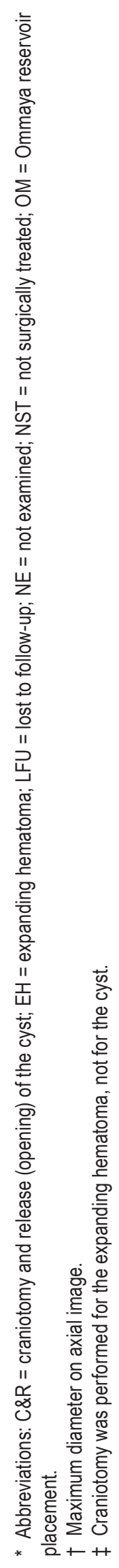




\section{Cyst formation following GKS}

traoperative findings, and histological findings of the cyst wall and associated granulomatous lesions. All patients underwent GKS, which was approved by the local ethics committee after written informed consent had been obtained from the patient or responsible relative.

\section{Results}

Chronic encapsulated expanding hematoma was associated with the cyst in 5 of the 18 patients (Table 1). Previous bleeding was observed in 7 patients. Axial imaging of the cyst in each case is shown in Fig. 1. The AVM nidus volume at the time of GKS ranged from 1.9 to $36 \mathrm{~cm}^{3}$ (median $6.1 \mathrm{~cm}^{3}$ ), and the prescription radiation dose at the nidus margin was 18-25 Gy (median 20 Gy). Complete obliteration of the nidus was achieved in 13 patients, and partial obliteration was obtained in 5 patients. Cyst formation was detected between 2.6 and 15 years (mean 7.0 years) after GKS. The median maximum size of the cyst on axial MR images was $39 \mathrm{~mm}$. Craniotomy was performed in 10 patients, including 2 patients in whom the incompletely obliterated nidus was removed at the same time, and an Ommaya reservoir was placed in 2 patients. Spontaneous regression of the cyst was observed in 1 patient. Serial MR imaging was performed in the other patients because the size of the cyst was stable or the cyst was asymptomatic. The contents of the cyst were analyzed in 4 patients (Cases 1, 5, 17, and 18); they were xanthochromic in 4 patients (Cases 1, 5, 13, and 18) and apparently hemorrhagic in 1 patient (Case 17). The mean protein concentration was $4397.9 \mathrm{mg} / \mathrm{dl}$ (range 2954-7263.5 mg/dl).

FIG. 1. Axial images of cysts obtained in each case.

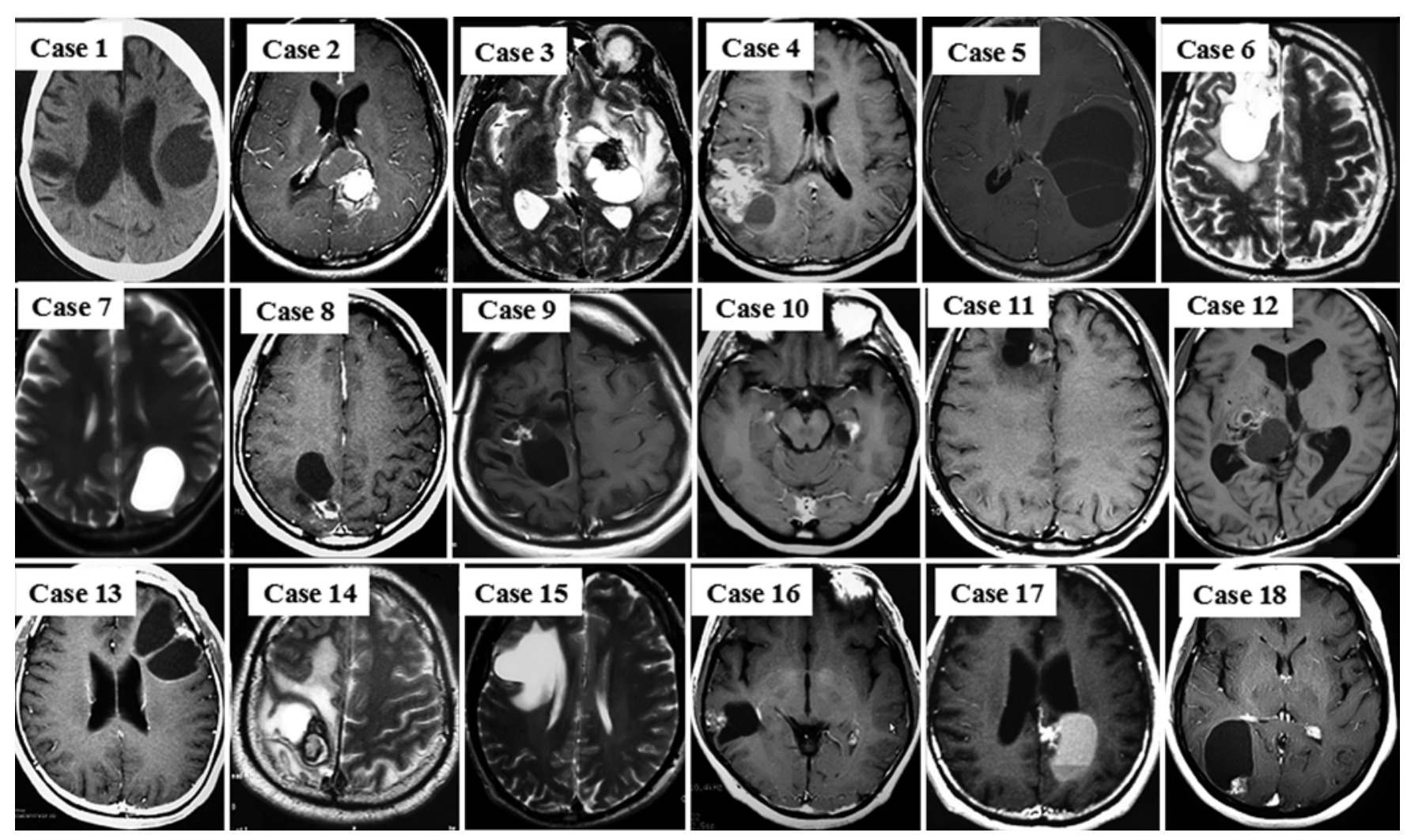

\section{Illustrative Cases}

Case 1. This 45-year-old man underwent GKS for a left frontotemporal AVM, which was treated with a radiation dose of 18 Gy to the lesion's margins. A cyst developed 13.5 years after GKS and gradually increased in size (Fig. 2). Left carotid angiography showed complete disappearance of the AVM nidus. The patient complained of right weakness and motor aphasia, and thus we placed an Ommaya reservoir with the aid of a local anesthetic agent (Fig. 2). The cyst contents were xanthochromic with a protein concentration of $3629 \mathrm{mg} / \mathrm{dl}$. After surgery, aspiration of the Ommaya reservoir was necessary 3 times, after which the cyst gradually decreased in size with improvement in the patient's neurological symptoms. Three years after surgery, this patient continues to participate in follow-up as an outpatient in good condition. This case demonstrates findings of intermittent intracystic hemorrhage and a high protein concentration in the cyst contents.

Case 13. This 27-year-old man underwent GKS for a left frontal AVM, accomplished using a margin dose of $20 \mathrm{~Gy}$. The patient was admitted to our hospital because he experienced general convulsions 5 years after GKS. Magnetic resonance imaging with Gd-GTPA enhancement revealed cyst formation associated with a nodular lesion (Fig. 3). The patient underwent cyst opening with removal of a reddish nodular lesion. The cyst contents were xanthochromic with a protein concentration of 2811 $\mathrm{mg} / \mathrm{dl}$. Histological examination of the cyst wall revealed 

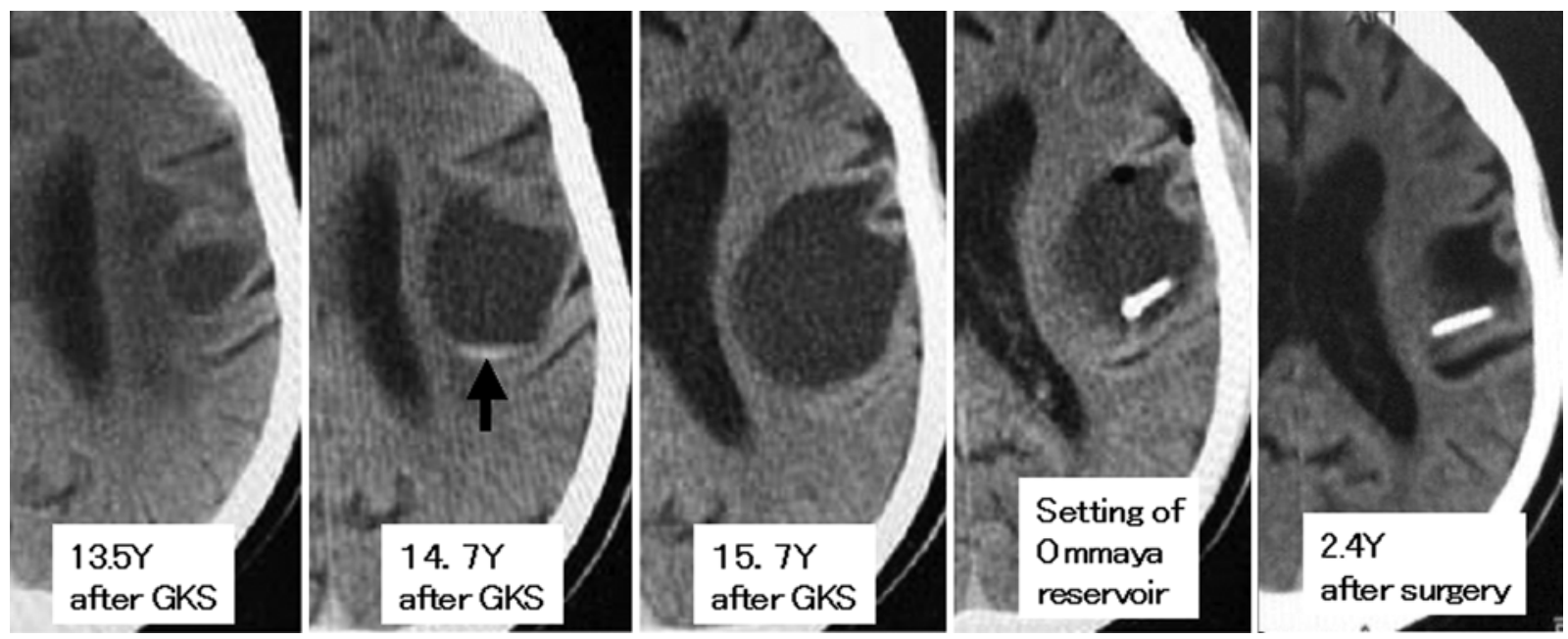

FIG. 2. Case 1. Computed tomography (CT) scans showing cyst formation 13.5 years after GKS. The cyst gradually enlarged, and after placement of an Ommaya reservoir the cyst gradually decreased in size. A CT scan obtained 14.7 years (Y) after GKS shows intracystic hemorrhage (arrow).

linear deposits of hemosiderin with gliosis, but no evidence of fresh hemorrhage in the cyst wall (Fig. 4 left). Histological examination of the nodular lesion demonstrated granulation tissue with chronic hemorrhage from newly developed capillary vessels (Fig. 4 right). This case demonstrates the possibility of intracystic hemorrhage from a nodular lesion that is enhanced by Gd-DTPA on MR images.

Case 17. This 40-year-old man underwent GKS for a callosal AVM using a margin dose of $20 \mathrm{~Gy}$. The patient was admitted to our hospital because he suffered from headache 5 years after GKS. Cyst formation associated with a nodular lesion was found on Gd-DTPA-enhanced MR images (Fig. 5). Intracystic hemorrhage was apparent. The patient underwent cyst opening with removal of the nodular lesion. The cyst contents were hemorrhagic, and histological examination of the cyst wall revealed deposits of hemosiderin with gliosis. Histological examination of the nodular lesion demonstrated hemorrhage from newly developed capillary vessels. This case illustrates chronic hemorrhaging into the cyst cavity from an angiomatous lesion.
Case 18. This 35-year-old woman underwent GKS with a margin dose of 25 Gy for a right occipital AVM. The patient was admitted to our hospital because she suffered from headache 12 years after GKS. Cyst formation with a nodular lesion was identified on Gd-DTPA-enhanced MR images (Fig. 6). The patient underwent cyst opening with removal of the nodular lesion. The cyst contents were xanthochromic with a high protein concentration $(2954 \mathrm{mg} / \mathrm{dl})$. Histological examination of the degenerated nidus revealed an endothelium that was markedly thickened, probably due to radiosurgery (Fig. 6). The reddish nodular angiomatous lesion contained capillary vessels with dilated sinusoid vessels compatible with a cavernous angioma. This case suggests the possibility of a radiation-induced cavernous angioma as a cause of intracystic hemorrhage.

\section{Discussion}

Pathologically, a cyst is a unilocular or multilocular epithelium-lined cavity that contains liquid or gaseous materials. Non-epithelium-lined cavities are classified as "pseudocysts." In clinical practice, both pseudocysts
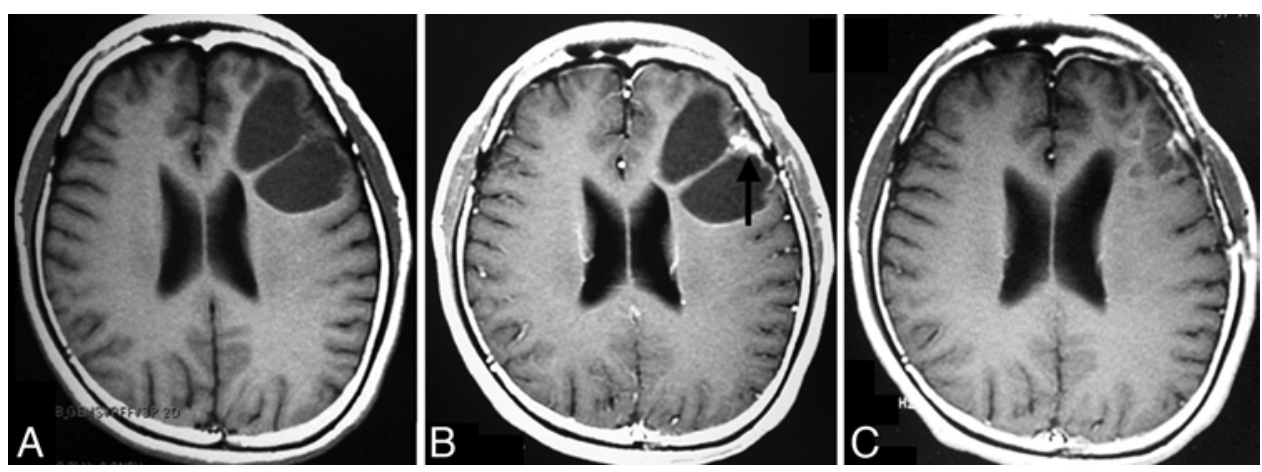

FiG. 3. Case 13. Magnetic resonance images obtained 5 years after GKS revealing a cyst in the left frontal lobe (A). A small nodular lesion was enhanced by injection of Gd-DTPA (B, arrow). After surgery, the cyst cavity disappeared completely (C). 


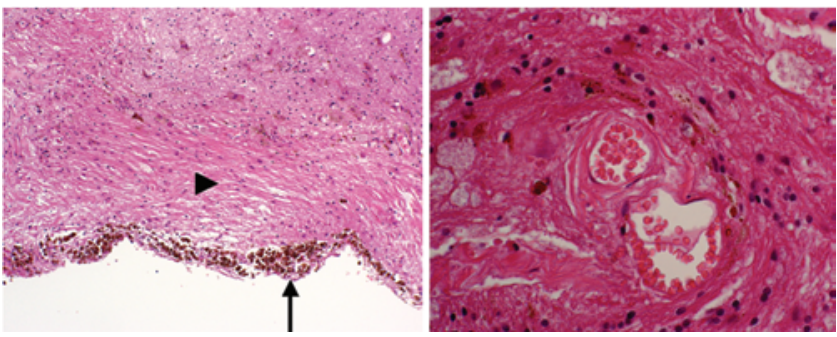

FIG. 4. Case 13. Left: Photomicrograph of the cyst wall revealing linear deposits of hemosiderin (arrow) with gliosis (arrowhead) but no evidence of fresh hemorrhage in the cyst wall. Right: Photomicrograph of the nodular lesion demonstrating inflammation, cell infiltration, and old hemorrhage, as well as granulation tissue with chronic hemorrhage from newly developed capillary vessels. H\&E, original magnification $\times 100$ (left) and $\times 400$ (right).

and cysts are generally referred to as "cysts." To be exact, a cystic cavity that develops after GKS is performed to treat an AVM should be called a "pseudocyst." For the sake of convenience, however, we use the term "cyst" in this study.

The exact mechanism of cyst formation remains unclear. We examined the dose-volume relationship in our series. Three patients harbored an AVM with a large nidus $\left(>20 \mathrm{~cm}^{3}\right)$, but the median volume in this series was $6.1 \mathrm{~cm}^{3}$, which was not so large. Cysts developed in patients with an AVM nidus that was relatively small, such as $1.9,2.4$, and $3.1 \mathrm{~cm}^{3}$. Finally, the median margin dose of $20 \mathrm{~Gy}$ is the standard dose used to treat an AVM, and we could not find any definite correlation between the dose-volume relationship and cyst development. Previous cases of cyst formation following GKS for an AVM are summarized in Table 2.5,7,9,16,17 In most of these series, the margin dose did not differ between all treated cases and cyst formation cases. The nidus volume at the time of GKS in the cases of cyst formation was relatively large in the series described by Pollock and Brown ${ }^{17}\left(15.2 \mathrm{~cm}^{3}\right)$ and Izawa et al. ${ }^{7}\left(9.9 \mathrm{~cm}^{3}\right)$. However, the nidus volume was not so large in the series described by Pan et al. ${ }^{16}$ $\left(3.9 \mathrm{~cm}^{3}\right)$ or in our series (median $6.1 \mathrm{~cm}^{3}$ ). Therefore, we think that a large nidus volume or a high radiation dose may be risk factors, but they are not essential. Pan et al. ${ }^{16}$ also found no correlation between cyst formation and AVM size or treatment dosage. We emphasize that cyst formation following GKS for AVM may occur even if the nidus is relatively small or the prescribed margin dose is not so high. Prior bleeding was found in 7 (38.9\%) of 18 patients. Bleeding before GKS was observed in 362 $(46.7 \%)$ of all 775 treated patients at our institution, and thus prior bleeding is also unlikely to be important in delayed cyst formation.

Increased permeability of injured blood vessels of the nidus, breakdown of the blood-brain barrier, and liquefaction of coagulation necrosis have all been suggested as possible causes of cyst formation. ${ }^{20}$ Our experience suggests that the initial stage of cyst formation is radiationinduced inflammation in the brain parenchyma around the irradiated nidus. This inflamed area is enhanced by Gd-DTPA on MR images. In Case 8 in this study, changes in the shape and size of the enhanced lesion were evident on MR images at the initial stage of cyst formation (Fig. 7). Extravasation of fluid from the inflammatory lesion results in the formation of a small cavity. Subsequently, in some cases this inflammation subsides and the cyst size decreases or stabilizes. On the other hand, angiomatous changes, such as the appearance of newly developed capillary vessels, may occur in the inflammatory lesion. Infiltration of inflammatory cells, granulomatous change, and chronic hemorrhage from newly developed capillary vessels were evident in our cases. These vessels are fragile and bleed repeatedly. Histological examination in Case 13 showed hemosiderin deposits on the cyst wall and fresh hemorrhage in the reddish nodular lesion. Repeated hemorrhage into the cyst cavity increased protein content and osmotic pressure, which caused the cyst to enlarge. Xanthochromic cyst contents with a high protein concentration were observed in our surgically treated cases, typically as shown in Cases 1 and 18. In Case 13 there was a very high protein concentration and deposits of hemosiderin on the surface of the cyst wall but not within the wall itself. These findings indicate that the origin of the hemorrhage was not the cyst wall. Previously, no evidence of neoplasia was found in cyst wall specimens. ${ }^{16}$

The increase in cyst size is mainly due to repeated hemorrhage from the angiomatous lesion into the cyst cavity. The appearance of nouveau formation on CT scans, as seen in Case 1 (Fig. 2), and findings of intracystic hemorrhage during surgery, as seen in Case 17, are also suggestive of this mechanism. Repeated hemorrhage
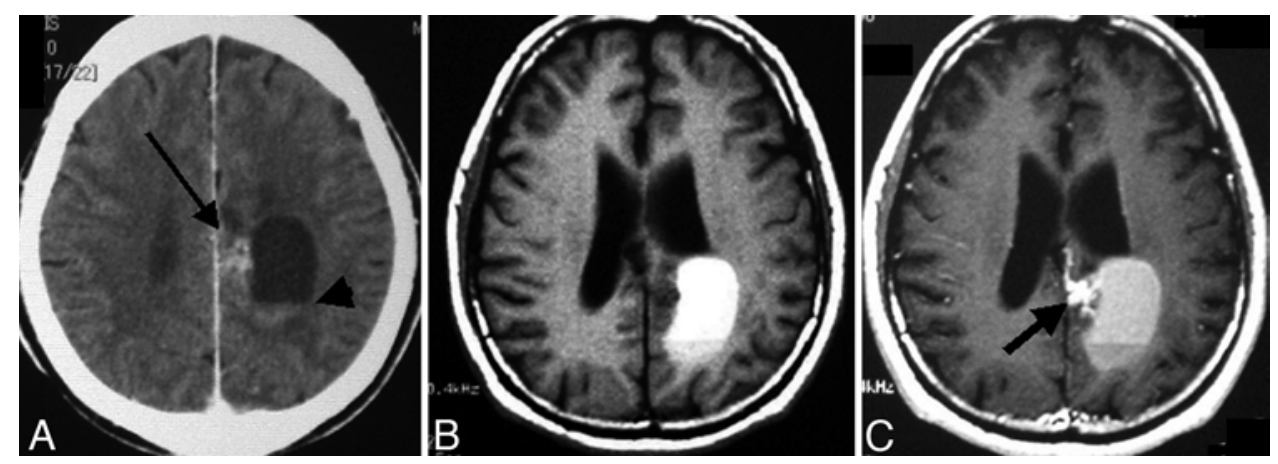

FIG. 5. Case 17. A: Contrast-enhanced CT scan showing a cyst in the left parietooccipital region with hemorrhage (arrowhead) and nodular enhancement (arrow). B and C: T1-weighted MR images revealing a high-intensity cyst suggesting hemorrhage $(B)$, and nodular enhancement following administration of GD-DTPA (C, arrow). 

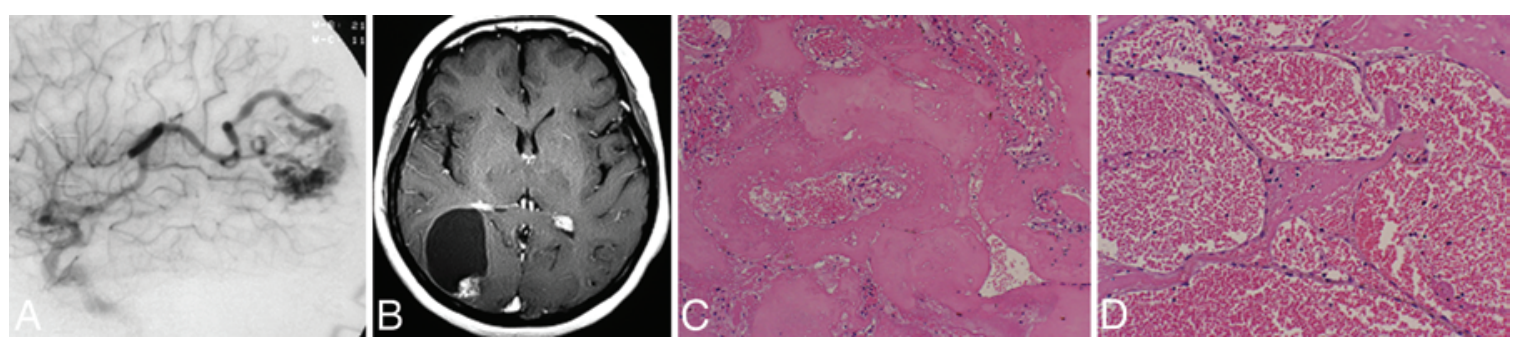

FIG. 6. Case 18. A: Right carotid angiogram showing an occipital AVM. B: A Gd-DTPA-enhanced MR image obtained 12 years after GKS was performed using a margin dose of $25 \mathrm{~Gy}$. The image demonstrates a large cyst with an enhanced nodular lesion in the right occipital lobe. C: Photomicrograph of the degenerated AVM nidus revealing markedly thickened endothelium, which was probably induced by radiosurgery. H\&E, original magnification $\times 100$. D: Photomicrograph of the reddish nodular angiomatous lesion showing capillary vessels with dilated sinusoid vessels compatible with a cavernous angioma. $\mathrm{H} \& \mathrm{E}$, original magnification $\times 400$.

from a degenerated irradiated nidus is also possible, but it is less likely because the angiographic and operative findings indicated complete occlusion of the nidus. In addition, an AVM associated with a cyst has been reported but that is rare. ${ }^{4,6}$

Magnetic resonance imaging detected nodular enhancement in 10 patients. In our early experience, Gdenhanced MR imaging was not used in the follow-up of patients with AVMs after GKS. Therefore, the occurrence of an angiomatous nodular lesion was not confirmed in 3 patients.

A reddish angiomatous nodular lesion was found and removed by surgery in Case 7, although Gd was not administered during preoperative MR imaging. The origin of this angiomatous lesion is unclear. An origin in the irradiated nidus is possible, but we think that the adjacent brain parenchyma is more likely, because the occurrence of an angiomatous lesion following GKS is not specific for an AVM. We had a case in which chronic encapsulated expanding hematoma developed within the brain parenchyma 11 years after GKS and radiotherapy for a dural arteriovenous fistula of the transverse-sigmoid sinus (Fig. 8). The operative findings indicated that a chronic encapsulated expanding hematoma was located under the arachnoid membrane and had developed in the brain parenchyma, with no continuity with the transverse-sigmoid sinus. From this case, we infer that radiation-induced angiomatous change after GKS is not specific to an AVM, and an angiomatous lesion has no continuity with the irradiated lesion in some cases. Based on our experience, a reddish nodular lesion, enhanced on MR imaging, is lo- cated between the occluded nidus and brain parenchyma, but does not lie entirely on the surface of the occluded nidus (Fig. 9). Therefore, we speculate that the angiomatous lesion develops within the brain parenchyma adjacent to the irradiated nidus. Figure 10 demonstrates a schematic drawing of the relationship between the nidus, angiomatous lesion, and cyst. If the angiomatous lesion were to develop within the degenerated nidus, all of the angiomatous lesion would be located on the surface of the nidus (Fig. 10 upper). However, we have no experience of such cases. If the angiomatous lesion develops in the brain parenchyma adjacent to the nidus, part of the angiomatous lesion is buried in the brain (Fig. 10 lower), as shown in Fig. 9.

If the hemorrhage from an angiomatous lesion spreads mainly into the brain parenchyma, a cyst develops and enlarges. On the other hand, if the hemorrhage occurs mainly within the angiomatous lesion, a so-called chronic encapsulated expanding hematoma develops. Therefore, a cyst with an angiomatous lesion and an expanding hematoma are essentially the same, and coexist in some cases. The histological examination of a chronic encapsulated expanding hematoma and the possibility of occurrence of cavernous angioma have been reported..$^{13}$ However, in our series, a cavernous angioma-like lesion was found in only one case (Case 18). Enhanced nodular lesions on MR images associated with a cyst may be of variable types such as radiation-induced cavernous angioma and non-cavernous angioma-like granulation tissue with newly developed fragile capillary vessels.

Predicting the future course of a cyst is very diffi-

TABLE 2: Previous cases of cyst formation following radiosurgery for AVMs*

\begin{tabular}{|c|c|c|c|c|c|c|}
\hline \multirow[b]{2}{*}{ Author \& Yr } & \multirow{2}{*}{$\begin{array}{c}\text { No. of Patients } \\
(\%)\end{array}$} & \multirow{2}{*}{$\begin{array}{l}\text { Mean or Median Age at } \\
\text { Cyst Formation (yrs) }\end{array}$} & \multicolumn{2}{|c|}{ Margin Dose (Gy) } & \multirow{2}{*}{$\begin{array}{l}\text { AVM Nidus Vol at Cyst } \\
\text { Formation }\left(\mathrm{cm}^{3}\right)\end{array}$} & \multirow{2}{*}{$\begin{array}{l}\text { Duration of Cyst } \\
\text { Formation (yrs) }\end{array}$} \\
\hline & & & All Cases & Cases W/ Cyst Formation & & \\
\hline Pollock \& Brown, 2001 & 6 (ND) & 24 & ND & median 16 & mean 15.2 & median 4 \\
\hline Pan et al., 2005 & 20 of $1353(1.5)$ & 31 & mean 19.8 & 20.7 & 3.9 & mean 8.2 \\
\hline Inoue, 2006 & 1 of $104(1.0)$ & ND & mean 19.5 & 20 & ND & 10 \\
\hline
\end{tabular}

\footnotetext{
* Abbreviation: ND = not described.
} 


\section{Cyst formation following GKS}

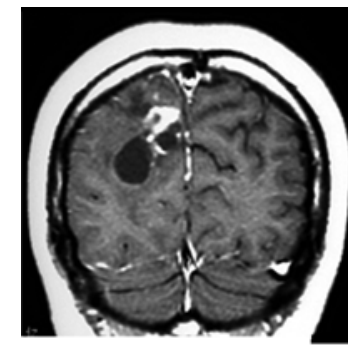

GKS 2.6Y

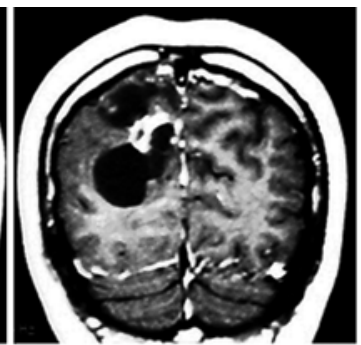

GKS 3.5Y

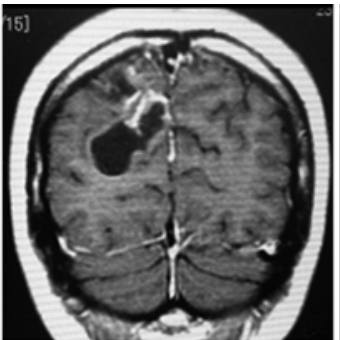

GKS $5 Y$

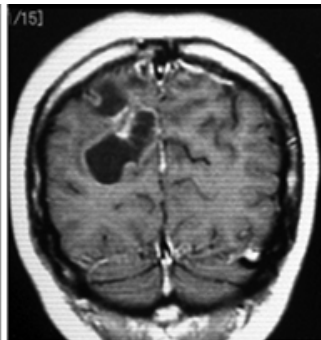

GKS $6 Y$

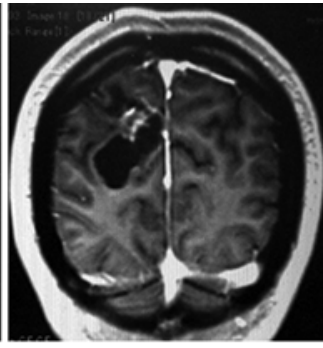

GKS 9Y

FIg. 7. Case 8. Serial coronal Gd-DTPA-enhanced MR images showing a cyst that developed 2.6 years after GKS. Both enhancement of the nodular lesion and the size of the cyst changed throughout the course of follow-up. Surgery was not performed because the cyst caused no symptoms.

cult. In our experience, some cysts become very large but remain asymptomatic or spontaneously decrease in size. In Case 8 a variety of changes appeared on follow-up images. Cyst formation developed 2.6 years after GKS, and the size of the cyst changed on follow-up imaging. In addition, the enhanced region around the cyst also changed. This transient enhancement is thought to represent inflammation rather than a developing angiomatous lesion.

We recommend regularly scheduled follow-up examinations for patients with asymptomatic cyst formation. If the cyst apparently increases in size and becomes symptomatic, surgery is indicated.${ }^{19}$ Placement of an Ommaya reservoir under local anesthesia is recommended for such cases..$^{10}$ Neurological symptoms tend to improve immediately after aspiration of the cyst content. Control of cyst growth is also obtained by repeat aspiration in most cases, although the long-term outcome remains unclear. Cystoperitoneal shunt insertion is also recommended. ${ }^{17}$ We placed an Ommaya reservoir in the patients in Cases 1 and 4, and cyst development was successfully controlled for at least 3 years (Case 1), although craniotomy for an associated expanding hematoma became necessary in Case 4. A series of 20 patients with delayed cyst formation included 6 who were symptomatic; 3 of these 6 patients received surgical treatment. ${ }^{16}$ Two underwent craniotomy, and an Ommaya reservoir was placed in another patient, which was later converted to a cystoperitoneal shunt. One of two patients with cyst formation underwent cyst fenestration by craniotomy. ${ }^{9}$ A less invasive cystoperitoneal shunt has been recommended for initial treatment, as is secondary cyst excision if the mass effect is not relieved by the cystoperitoneal shunt. ${ }^{17}$ However, the presence of an enhanced nodular angiomatous lesion on Gd-enhanced MR images was not discussed in these series. As we have suggested, delayed cyst formation becomes symptomatic due to a mass effect, which results from repeated minor hemorrhage from the angiomatous lesion. Therefore, we believe that craniotomy and wide opening of the cyst with removal of the angiomatous lesion is essential for the treatment of a cyst associated with an angiomatous lesion. Placement of an Ommaya reservoir in the opened cyst cavity will then not be necessary. Endoscopic surgery with cyst fenestration and removal of an angiomatous lesion may be useful for a deep-seated or multilobular cyst. Our proposed treatment algorithm is summarized in Fig. 11.

\section{Conclusions}

Cyst formation is clinically important as a late complication following GKS for an AVM. This complication should be taken into consideration during follow-up of patients. Cysts developing after GKS for an AVM enlarge mainly because of repeated minor hemorrhage from a reddish nodular angiomatous lesion that develops within an adjacent brain area. This indicates that the optimal treatment is wide opening of the cyst with removal of the associated angiomatous lesion by craniotomy.
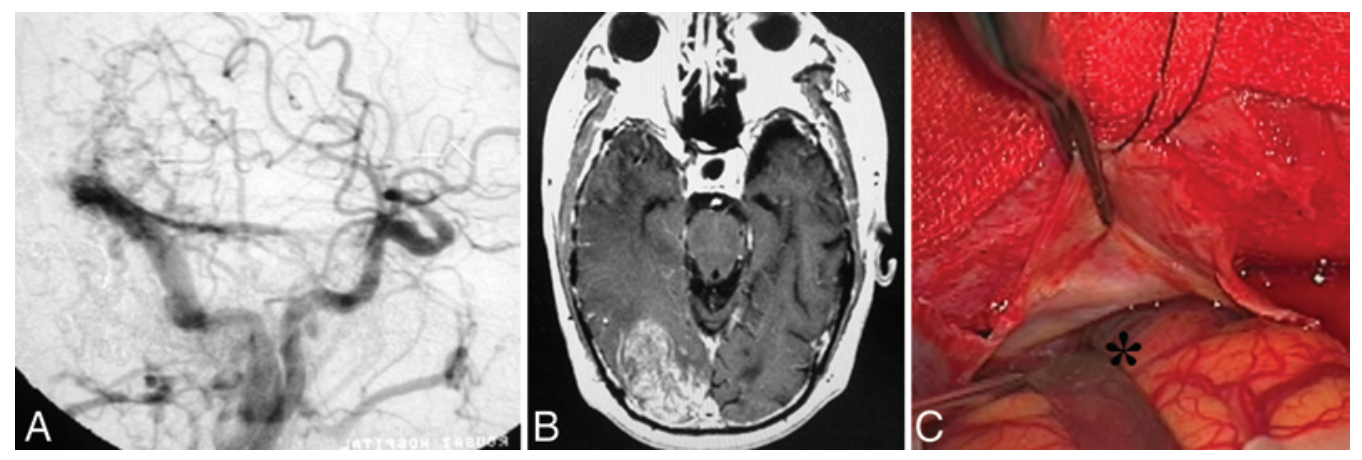

Fig. 8. A: Right carotid angiogram showing a dural arteriovenous fistula of the transverse-sigmoid sinus. B: An MR image obtained 11 years after GKS and radiotherapy revealing a chronic encapsulated expanding hematoma in the right occipital lobe. C: Intraoperative photograph showing no direct continuity between the dura mater and the expanding hematoma. Asterisk indicates the expanding hematoma. 

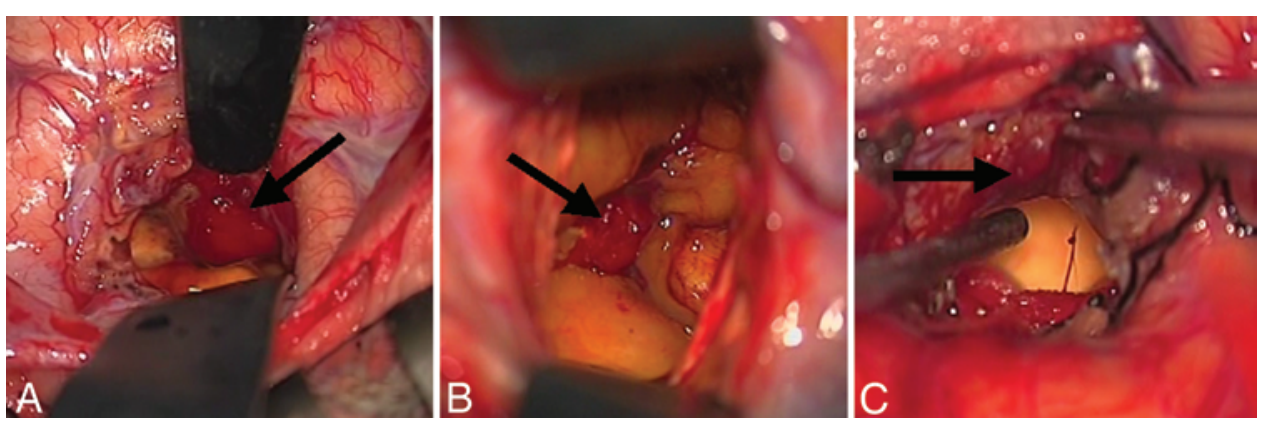

FIG. 9. Intraoperative photographs from Cases 18 (A), 17 (B), and 16 (C) showing that the cyst wall is brown. Reddish nodular angiomatous lesions (arrows) were easily identified buried in the brain parenchyma adjacent to the occluded AVM nidus.

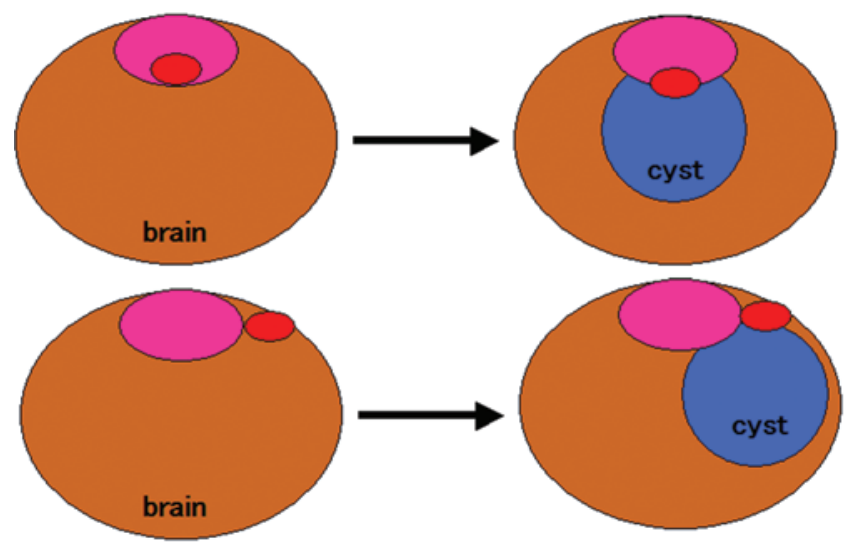

FIG. 10. Schematic drawings of the relationship between the AVM nidus (pink oval), angiomatous lesion (red oval), and cyst (blue circle). Upper: If an angiomatous lesion develops in the degenerated AVM nidus, all of the angiomatous lesion is located on the surface of the nidus. Lower: If an angiomatous lesion develops in the brain parenchyma adjacent to the irradiated nidus, part of the angiomatous lesion is buried in the brain.

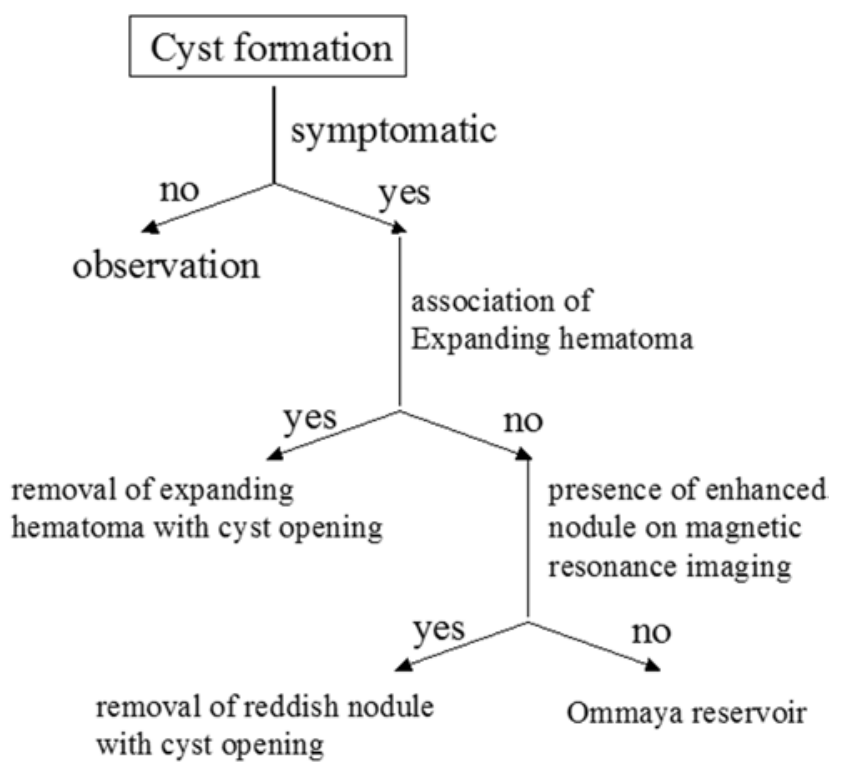

FIG. 11. The proposed treatment algorithm for cyst formation following GKS for AVM.

\section{Disclosure}

The authors report no conflict of interest concerning the materials or methods used in this study or the findings specified in this paper.

Author contributions to the study and manuscript preparation include the following. Conception and design: Shuto. Acquisition of data: all authors. Analysis and interpretation of data: Shuto. Drafting the article: Shuto. Critically revising the article: all authors. Reviewed submitted version of manuscript: all authors. Approved the final version of the manuscript on behalf of all authors: Shuto. Statistical analysis: Shuto. Administrative/technical/material support: Ohtake, Matsunaga. Study supervision: Shuto.

\section{References}

1. Flickinger JC, Kondziolka D, Pollock BE, Maitz AH, Lunsford LD: Complications from arteriovenous malformation radiosurgery: multivariate analysis and risk modeling. Int J Radiat Oncol Biol Phys 38:485-490, 1997

2. Ganz JC, Reda WA, Abdelkarim K, Hafez A: A simple method for predicting imaging-based complications following gamma knife surgery for cerebral arteriovenous malformations. J Neurosurg 102 (Suppl):4-7, 2005

3. Guo WY, Lindquist C, Karlsson B, Kihlström L, Steiner L: Gamma knife surgery of cerebral arteriovenous malformations: serial MR imaging studies after radiosurgery. Int J Radiat Oncol Biol Phys 25:315-323, 1993

4. Hatashita S, Tajima A, Takeuchi K, Ueno H: Arteriovenous malformation associated with a large cyst-case report. Neurol Med Chir (Tokyo) 35:377-379, 1995

5. Inoue HK: Long-term results of Gamma Knife surgery for arteriovenous malformations: 10- to 15-year follow up in patients treated with lower doses. J Neurosurg 105 Suppl: 64-68, 2006

6. Itakura T, Takifuji K, Ozaki F, Itatani K, Nishiguchi T, Hayashi S, et al: Cystic arteriovenous malformation. A case report. Acta Neurochir (Wien) 96:154-158, 1989

7. Izawa M, Hayashi M, Chernov M, Nakaya K, Ochiai T, Murata $\mathrm{N}$, et al: Long-term complications after gamma knife surgery for arteriovenous malformations. J Neurosurg 102 Suppl: 34-37, 2005

8. Kaido T, Hoshida T, Uranishi R, Akita N, Kotani A, Nishi N, et al: Radiosurgery-induced brain tumor. Case report. J Neurosurg 95:710-713, 2001

9. Kano H, Lunsford LD, Flickinger JC, Yang HC, Flannery TJ, Awan NR, et al: Stereotactic radiosurgery for arteriovenous malformations, Part 1: management of Spetzler-Martin Grade I and II arteriovenous malformations. Clinical article. J Neurosurg 116:11-20, 2012

10. Kim MS, Lee SI, Sim JH: A case of very large cyst formation with Gamma Knife radiosurgery for an arteriovenous malfor- 


\section{Cyst formation following GKS}

mation. Stereotact Funct Neurosurg 72 (Suppl 1):168-174, 1999

11. Kurita H, Sasaki T, Kawamoto S, Taniguchi M, Kitanaka C, Nakaguchi $\mathrm{H}$, et al: Chronic encapsulated expanding hematoma in association with gamma knife stereotactic radiosurgery for a cerebral arteriovenous malformation. Case report. J Neurosurg 84:874-878, 1996

12. Maruyama K, Kawahara N, Shin M, Tago M, Kishimoto J, Kurita $\mathrm{H}$, et al: The risk of hemorrhage after radiosurgery for cerebral arteriovenous malformations. N Engl J Med 352: 146-153, 2005

13. Motegi H, Kuroda S, Ishii N, Aoyama H, Terae S, Shirato H, et al: De novo formation of cavernoma after radiosurgery for adult cerebral arteriovenous malformation-case report. Neurol Med Chir (Tokyo) 48:397-400, 2008

14. Nakamizo A, Suzuki SO, Saito N, Shono T, Matsumoto K, Onaka S, et al: Clinicopathological study on chronic encapsulated expanding hematoma associated with incompletely obliterated AVM after stereotactic radiosurgery. Acta Neurochir (Wien) 153:883-893, 2011

15. Ogilvy CS, Stieg PE, Awad I, Brown RD Jr, Kondziolka D, Rosenwasser R, et al: AHA Scientific Statement: Recommendations for the management of intracranial arteriovenous malformations: a statement for healthcare professionals from a special writing group of the Stroke Council, American Stroke Association. Stroke 32:1458-1471, 2001

16. Pan HC, Sheehan J, Stroila M, Steiner M, Steiner L: Late cyst formation following gamma knife surgery of arteriovenous malformations. J Neurosurg 102 Suppl:124-127, 2005
17. Pollock BE, Brown RD Jr: Management of cysts arising after radiosurgery to treat intracranial arteriovenous malformations. Neurosurgery 49:259-265, 2001

18. Pollock BE, Gorman DA, Coffey RJ: Patient outcomes after arteriovenous malformation radiosurgical management: results based on a 5- to 14-year follow-up study. Neurosurgery 52:1291-1297, 2003

19. Shuto T, Matsunaga S, Suenaga J: Surgical treatment for late complications following gamma knife surgery for arteriovenous malformations. Stereotact Funct Neurosurg 89:96102,2011

20. Yamamoto M, Jimbo M, Hara M, Saito I, Mori K: Gamma knife radiosurgery for arteriovenous malformations: longterm follow-up results focusing on complications occurring more than 5 years after irradiation. Neurosurgery 38:906914, 1996

Manuscript submitted February 9, 2012.

Accepted June 5, 2012.

Please include this information when citing this paper: DOI: 10.3171/2012.6.GKS12318.

Address correspondence to: Takashi Shuto, M.D., Department of Neurosurgery, Yokohama Rosai Hospital, 3211 Kozukue-cho, Kouhoku-ku, Yokohama, Kanagawa 222-0036, Japan. email: shuto@ yokohamah.rofuku.go.jp. 